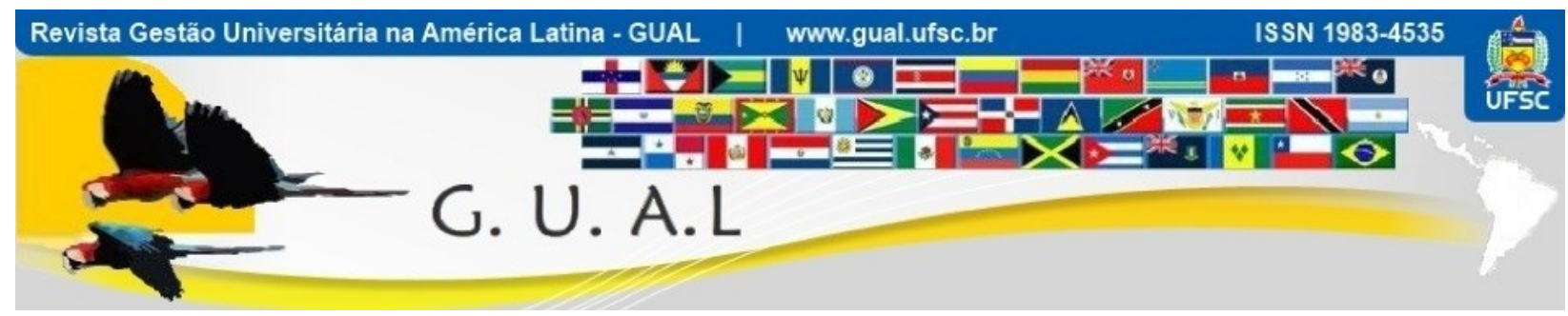

DOI: http://dx.doi.org/10.5007/1983-4535.2017v10n3p204

\title{
DESEMPENHO NO ENADE E SUA RELAÇÃO COM A AVALIAÇÃO DOCENTE PELO DISCENTE
}

\section{PERFORMANCE IN ENADE AND ITS RELATION TO THE STUDENT TEACHER EVALUATION}

Maria Elisabeth Pereira Kraemer, Mestre Universidade do Vale do Itajaí - UNIVALI kraemer@univali.br

Miguel Angel Verdinelli, Doutor Universidade do Vale do Itajaí - UNIVALI nupad@univali.br

Recebido em 14/fevereiro/2016

Aprovado em 14/junho/2017

Sistema de Avaliação: Double Blind Review

Esta obra está sob uma Licença Creative Commons Atribuição-Uso. 


\title{
RESUMO
}

Este artigo tem por objetivo analisar a relação entre os desempenhos docente e discente nos processos de avaliação interna e externa do Curso de Administração da UNIVALI de Itajaí. A população do estudo na avaliação externa foi composta de 232 alunos concluintes do Curso de Administração que participaram do ENADE em 2012. A análise foi feita mediante relatório disponibilizado no site do INEP. Na avaliação interna, foram selecionados 38 professores das respectivas disciplinas envolvidas em cada questão. Utilizaram-se os boletins de desempenho de cada professor, gerados pela Avaliação Institucional, os quais destacam a ação docente e contêm ainda as médias aritméticas obtidas em cada critério de desempenho avaliado: Domínio Técnico Científico (DTC), Habilidades de Ensino (HE) e Desenvolvimento Pessoal e Situacional (DPS). Para a análise estatística foram utilizados os métodos paramétricos e não paramétricos, ou seja, o coeficiente paramétrico de correlação de Pearson e o coeficiente não paramétrico por postos ou ranking de Spearman. Como as amostras eram pequenas, o teste usado foi o de Shapiro-Wilk. Os resultados obtidos indicam que o desempenho dos alunos e a dimensão DTC possuem distribuição normal $(\mathrm{p}>\alpha)$, o que possibilitou usar o coeficiente de Pearson. Já as dimensões Habilidade de Ensino (HE) e Desempenho Pessoal e Situacional (DPS) não têm distribuições normais $(\mathrm{p}<\alpha)$, obrigando a usar o coeficiente de Spearman. As Fig.1, 2 e 3 mostram graficamente a falta de relação entre a avaliação docente (interna) e o resultado dos alunos no ENADE (avaliação externa).

Palavras chave: Gestão Universitária. Avaliação Discente. Avaliação Docente.

\begin{abstract}
This article aims to analyze the relationship between teacher and student performance in internal and external evaluation processes Administration Course UNIVALI of Itajaí. The study population in the external evaluation was composed of 232 students graduating Administration Course attending the ENADE in 2012. The analysis was done by INEP report made available on the site. In internal evaluation, we selected 38 teachers of the respective disciplines involved in eachissue. The performance reports of each teacher were used, generated by the Institutional Assessment, which highlight the teaching action and still contain the averages obtained for each evaluated performance criterion: Domain Scientific Technical (DTC), Teaching Skills (HE) and Personal Development and Situational (DPS). For statistic alanalysis, the parametric and non-parametric methods were used, namely parametric Pearson correlation coefficient and the coefficient station sor by non-parametric Spearmanrank. As the samples were small, the test used wasthe Shapiro-Wilk test. The result sindicate that the performance of student sand the DTC sizehave normal distribution $(p>\alpha)$ which made it possible to use the Pearson coefficient. Have dimensions Teaching Ability (HE) and Performance Personal and Situational (DPS) do not have normal distributions ( $p$ $<\alpha$ ), forcing using the Spearman coefficient. The Fig.1, 2 and 3 show graphically the lack of relationship between teach erassessment (internal) and the result of students in ENADE (external evaluation).
\end{abstract}

Keywords: University Management .Studen tevaluation .Teacher evaluation 


\section{INTRODUÇÃO}

O Exame Nacional de Desempenho dos Estudantes - ENADE é aplicado aos estudantes que preenchem os critérios estabelecidos pela legislação vigente. Seu objetivo é aferir o rendimento dos alunos dos cursos de graduação em relação aos conteúdos programáticos, as habilidades e competências para a permanente atualização profissional, aos conhecimentos referentes à realidade brasileira e mundial e a outras áreas do conhecimento. É um exame periódico, aplicado nacionalmente de 3 em 3 anos (o ciclo avaliativo do SINAES é trienal) em cada curso e constitui-se um componente curricular obrigatório dos cursos de graduação.

O ENADE é composto pela prova, o questionário de Avaliação Discente da Educação Superior, o questionário dos coordenadores de cursos e a percepção do aluno sobre a prova.

A prova é constituída por 40 questões, sendo: 10 questões de Formação Geral (8 de múltipla escolha e 2 questões discursivas) e 30 questões do Componente Específico (27 questões de múltipla escolha e 3 questões discursivas). Os conteúdos de formação geral têm peso de $25 \%$ para compor a nota no ENADE e os conteúdos específicos de $75 \%$.

Assim como todas as IES do Sistema Federal de Educação Superior (Público e Privado), a UNIVALI também participa do ENADE.

Considerada uma das maiores instituições de ensino superior do Brasil, a UNIVALI está localizada no litoral centro-norte de Santa Catarina. Sua estrutura multicampi faz com que esteja presente nas cidades de Itajaí, Balneário Camboriú, Biguaçu, Florianópolis, Piçarras, São José e Tijucas.

Com sede em Itajaí, o Curso de Administração, atento às perspectivas de ampliação de seu mercado de trabalho tem como diferencial a sua integração com as empresas da região na busca da qualidade dos gestores e do processo de gestão organizacional.

A análise da relação entre a qualidade e a satisfação com o ensino de graduação a partir de indicadores externos e internos, poderão servir de base ao planejamento de ações integradas que poderiam oportunizar a construção da qualidade como um processo contínuo e aberto, mediante o qual, todos os setores da universidade e as pessoas envolvidas repensem objetivos e operacionalizem ações articuladas.

Assim, pretende-se juntar as informações do ENADE e da AI para coordenar, acompanhar e analisar comparativamente as modalidades de avaliação e seus resultados, a fim 
de encaminhar ações em parceria com os gestores dos cursos, contemplando, dessa forma, a política institucional, numa ação participativa e compartilhada.

Diante desses fatores, este trabalho se propõe a verificar: Qual a relação entre o desempenho docente e discente nos processos de avaliação interna e externa do curso de administração da UNIVALI de Itajaí?

Para responder a essa questão de pesquisa, foi definido o seguinte objetivo: Analisar a relação entre o desempenho docente e discente nos processos de avaliação interna e externa do curso de administração da UNIVALI de Itajaí.

Portanto, a contribuição do presente estudo consubstancia-se na realização de uma análise das relações entre o desempenho do ENADE com o desempenho docente, propiciando uma ligação entre a avaliação externa e a avaliação interna, o que viria a auxiliar no processo de tomada de decisão e subsidiar o estabelecimento de metas e ações a serem atingidas nos projetos institucionais.

\section{SISTEMA NACIONAL DE AVALIAÇÃO DA EDUCAÇÃO SUPERIOR (SINAES)}

Criado pela Lei $\mathrm{n}^{\circ}$ 10.861, de 14 de abril de 2004, o Sistema Nacional de Avaliação da Educação Superior (SINAES) é formado por três componentes principais: a avaliação das instituições, dos cursos e do desempenho dos estudantes. O SINAES avalia todos os aspectos que giram em torno desses três eixos: o ensino, a pesquisa, a extensão, a responsabilidade social, o desempenho dos alunos, a gestão da instituição, o corpo docente, as instalações e vários outros aspectos. (BRASIL 2004b)

Ele possui uma série de instrumentos complementares: autoavaliação, avaliação externa, ENADE, Avaliação dos cursos de graduação e instrumentos de informação (censo e cadastro).

Neste trabalho abordar-se-á o ENADE, pois seu foco é sua relação com a avaliação docente.

\subsection{EXAME NACIONAL DE DESEMPENHO DOS ESTUDANTES (ENADE)}

O Exame Nacional de Desempenho dos Estudantes (ENADE) é um dos procedimentos de avaliação do Sistema Nacional de Avaliação da Educação Superior (SINAES). É realizado pelo Instituto Nacional de Estudos e Pesquisas Educacionais Anísio Teixeira (INEP), autarquia vinculada ao Ministério da Educação (MEC), segundo diretrizes estabelecidas pela 
Comissão Nacional de Avaliação da Educação Superior (CONAES), órgão colegiado de coordenação e supervisão do SINAES.

Destaca-se que o ENADE é componente curricular obrigatório dos cursos de graduação, sendo o registro de participação condição indispensável para a emissão do histórico escolar, independentemente de o estudante ter sido selecionado ou não no processo de amostragem do INEP. (INEP, 2012).

A partir de 2012 somente os concluintes fizeram a prova, pois a mesma era aplicada para os ingressantes (estudantes que tenham iniciado o curso a ser avaliado pelo ENADE 2012 com matrícula inicial no ano letivo de 2012 em curso a ser avaliado pelo ENADE) e concluintes (estudantes que tenham expectativa de conclusão do curso a ser avaliado pelo ENADE 2012 até julho de 2013, assim como aqueles que tenham completado mais de 80\% (oitenta por cento) da carga horária do curso até o período final de inscrição).

Os concluintes que não realizarem a prova não colarão grau enquanto não regularizarem a sua situação junto ao INEP, devendo aguardar nova edição do exame cuja periodicidade é trienal ou ser inscrito como aluno irregular no ano seguinte (INEP, 2012).

O Conceito do ENADE é calculado para cada curso, tendo como unidade de observação a instituição de ensino superior - IES, o município da sede do curso e a área de avaliação e é apresentado em cinco categorias ( 1 a 5), sendo que 1 é o resultado mais baixo e 5 é o melhor resultado possível, na área.

As publicações dos resultados do ENADE são feitos mediante sete relatórios, conforme apresentado no site do INEP (2014): "relatório do aluno, relatório do curso, relatório da área, relatório da instituição, resumo técnico, relatório de conceitos e relatório técnico-científico". Esses relatórios apresentam os resultados obtidos no ENADE e deverão contribuir para o aperfeiçoamento dos processos de ensino aprendizagem e das condições de ensino e do próprio sistema de avaliação dos cursos de graduação.

\section{AVALIAÇÃO INSTITUCIONAL NA UNIVALI}

O movimento da Universidade do Vale do Itajaí UNIVALI em prol da implantação da Avaliação Institucional foi marcado, nos anos de 1993 e 1994, por uma importante ação: a constituição da Comissão de Avaliação Institucional - COAVI, junto à Pró-Reitoria de Ensino (ProEn), que quando foi criados os Centros de Educação na UNIVALI (junho de 1998), passou a ter o status de Coordenadoria de Avaliação Institucional. (UNIVALI, 2010). 
Após estudos sobre experiências de avaliação de outras universidades a Comissão de Avaliação Institucional, promoveu em 1994 um diagnóstico geral de tipo qualitativo e quantitativo aplicado a 26 cursos de graduação dos Campi Itajaí, Balneário Camboriú, Tijucas e Biguaçu, o qual possibilitou a avaliação dos docentes pelos discentes e vice-versa. Também realizou o $1^{\circ}$ Seminário Estadual de Avaliação Institucional, juntamente com a Associação Catarinense de Fundações Educacionais ACAFE, sendo publicados os respectivos Anais. Com esses eventos foi marcado o ingresso da UNIVALI no PAIUB.

Nos anos de 1995 a 2003, foram submetidos à avaliação:o Ensino Fundamental e Médio do Colégio de Aplicação (Unidades de Itajaí e Tijucas); o currículo, a formação geral e a infraestrutura dos cursos de graduação pelo Egresso; a Prática Forense do Curso de Direito; a Prática Desportiva;19 cursos de pós-graduação lato sensu;o perfil socioeconômico e cultural dos alunos da graduação (perfil discente);o curso de pós-graduação stricto sensu em Ciência Jurídica;o Estágio Supervisionado dos Cursos de Direito e do Centro de Ciências da Saúde; o desempenho das funções institucionais, gerenciais, acadêmicas e políticas das coordenações de curso. (UNIVALI, 2010).

Em 2004 foi realizada a Avaliação Institucional do Hospital Universitário Pequeno Anjo. Nesse mesmo ano ocorreu a terceira edição do Perfil dos Estudantes de Graduação: questionários on-line foram aplicados e analisados as respostas de 11.103 estudantes, 51,0\% do total dos matriculados na UNIVALI; os resultados desse levantamento estão publicados no documento Perfil dos Estudantes de Graduação da Universidade do Vale do Itajaí (UNIVALI, 2005).

No primeiro semestre do ano seguinte, a $11^{\mathrm{a}}$ edição da Avaliação Institucional realizou a avaliação de todos os cursos de graduação e sequenciais.

Em 2007, foi realizada a $13^{\mathrm{a}}$ edição da Avaliação Institucional, sendo diferente das anteriores, pois os indicadores foram remodelados nos cursos de graduação passando agora serem agrupados em três dimensões: infraestrutura e serviços do campus, curso de graduação e disciplinas regulares.

Já em 2008 na $14^{\mathrm{a}}$ edição foram feitas também novas modificações. Na dimensão infraestrutura e serviços do campus foram acrescidos: Ouvidoria, Central de Atendimento, Secretarias Acadêmicas, Setor de Bolsas, Meios de Comunicação, Atendimento da Biblioteca, Serviços on-line e prestação de serviços. 
Em 2009 teve a 15 a edição cujo resultado a Pró-Reitoria de Ensino deu continuidade ao processo sistemático de geração de informações para o planejamento e a tomada de decisão. (UNIVALI, 2010).

$\mathrm{O}$ processo de avaliação institucional tem possibilitado: fornecer subsídios à elaboração dos Relatórios de Autoavaliação da Univali, pela CPA; oferecer importante contribuição à troca de experiências entre as Instituições de Ensino Superior do Sistema ACAFE, para a construção de um encaminhamento comum, com vistas à sistematização da avaliação institucional em suas unidades; utilização de desempenho por ocasião de processos seletivos internos e incorporação ao atual Plano de Carreira, Sucessão e Remuneração. (UNIVALI, 2010).

Hoje, nos Cursos Presenciais de Graduação, a Avaliação Institucional está em sua 23 ${ }^{a}$ edição em 2014/II, nos Cursos de Graduação em EAD em sua 10ª em 2014/II, no Colégio de Aplicação em sua $15^{\mathrm{a}}$ edição em 2014/II e nos Cursos de Mestrado e Doutorado em sua $10^{\mathrm{a}}$ em 2014/II.

E assim todos os anos a Avaliação Institucional vem sendo usada pela Universidade UNIVALI como instrumento de gestão e de autoconhecimento.

Em seu Projeto Institucional, a Universidade tem como meta o rigor com a qualidade de ensino; está consciente, portanto, da necessidade de um processo de avaliação permanente, criterioso e adequado às políticas nacionais. Com a promulgação da Lei $\mathrm{n}^{0} 10.861$, de 14 de abril de 2004, que instituiu o Sistema Nacional de Avaliação da Educação Superior SINAES, a UNIVALI deu continuidade ao aperfeiçoamento de seu Programa de Avaliação Institucional, ampliando-o para diferentes modalidades.

\subsection{AVALIAÇÃO DOS CURSOS DE GRADUAÇÃO}

Constitui o foco principal do processo de avaliação; é composta pelas seguintes variáveis: “Avaliação do desempenho docente", que tem por objetivo identificar a qualidade da ação dos professores e os aspectos que podem ser melhorados pelos docentes e pela Universidade; "Avaliação discente pelo docente", que procura identificar e analisar o desempenho e envolvimento dos alunos no processo ensino/aprendizagem; "Autoavaliação docente", que busca diagnosticar o perfil dos professores da instituição e a dinâmica interna da atividade pedagógica; "autoavaliação discente", que visa indicar o desempenho dos estudantes e sua atuação em sala de aula e a "Avaliação da infraestrutura do curso", em que se avaliam as condições oferecidas pelo curso para o desenvolvimento das atividades de 
ensino/aprendizagem, o material didático utilizado, as condições de acesso e uso da biblioteca, as atividades de extensão e/ou de pesquisa, o ambiente físico de sala de aula e o sistema de comunicação.

Como o enfoque deste estudo é o Desempenho no ENADE e sua relação com a avaliação docente pelo discente, dar-se-á destaque agora na avaliação do desempenho docente.

\subsection{AVALIAÇÃO DO DESEMPENHO DOCENTE}

A avaliação do desempenho sempre existiu em toda carreira acadêmica, principalmente antes da obtenção da estabilidade e por ocasião de tomada de decisões referentes a promoções e à concessão de estabilidade. Em muitas instituições, porém, tendo obtido a estabilidade e atingido o nível mais alto que aspira o professor não volta a sofrer uma avaliação formal (ORMROD, 1986)

Vários trabalhos têm sido escritos sobre a definição e classificação do trabalho dos docentes do Ensino Superior, por exemplo, os de Boyer(1990), Bowen e Schuster(1986) e Rhodes(1990).

Pesquisas extensivas sobre vários aspectos a respeito do uso das respostas dos alunos para avaliar o desempenho do professor têm contribuído grandemente para a compreensão do processo ensino-aprendizagem.

A avaliação de docentes tem como objetivo melhorar o desempenho do corpo docente (função formativa); embasar decisões equitativas e eficientes com referência ao corpo docente (função somativa).

Sua função para a instituição é coletar evidências sobre o desempenho do professor, para tomar decisões sobre a efetivação, renovação de contratos e promoção; verificar se os objetivos institucionais estão sendo atingidos pelos docentes. Para os docentes seu uso é de coletar informações que lhes possibilitem melhorar seu ensino; identificar seus pontos fortes e fracos; refletir sobre sua atuação; verificar qual é a opinião dos alunos sobre seu desempenho. (BLAKBURN; PUTTEN, 1998)

Para Reifschneider (2008) a avaliação de desempenho docente pode ter uma concepção formativa que busca a melhora de desempenho futuro ou a concepção somativa em que se avalia o passado.

\section{MATERIAL E MÉTODO}


Para melhor aprender o fenômeno em estudo, foi traçado caminhos metodológico para o desenvolvimento desta pesquisa. Portanto, a seguir é explicitado: a abordagem da pesquisa, a população e variáveis pesquisadas e o relacionamento entre a avaliação externa e interna.

\subsection{ABORDAGEM DA PESQUISA}

Esta pesquisa teve como objetivo analisar a relação entre o desempenho docente e discente nos processos de avaliação externa e interna do curso de Administração da UNIVALI de Itajaí. Para cumprir este objetivo foram aplicadas pesquisas de análise documental e descritivas de abordagem quali-quantitativa.

Quanto aos procedimentos técnicos, a pesquisa foi do tipo documental, na qual se recorre a fontes mais diversificadas e dispersas, sem tratamento analítico. (FONSECA, 2002).

Do ponto de vista dos seus objetivos, a pesquisa foi descritiva, a qual visa descrever as características de determinada população ou fenômeno ou oestabelecimento de relações entre variáveis.Envolve o uso de técnicas padronizadas de coleta de dados: questionário eobservação sistemática.Assume, em geral, a forma de levantamento ou survey. (BABBIE, 2001).

Quanto à abordagem do problema, a pesquisa foi quantitativa, o que significa traduzir em números opiniões e informações para classificá-las e analisá-las. Requer o uso de recursos e de técnicas estatísticas (percentagem, média, moda, mediana, desvio-padrão, coeficiente de correlação, análise de regressão, etc.) (POLIT; BECKER; HUNGLER, 2004).

Esta pesquisa também foi qualitativa, o que, para Bogdan e Biklen (1994) é um conjunto dinâmico e aberto de afirmativas, concepções, proposições sistematicamente relacionadas para nortear tal proposta e, por conseguinte, o entendimento do pesquisador acerca do que está sendo pesquisado.

\subsection{POPULAÇÃO E VARIÁVEIS PESQUISADAS}

A população do estudo, quanto à avaliação externa, foi composta de 232 alunos concluintes do curso de Administração da Univali do campus de Itajaí, que participaram do ENADE. A partir dos resultados das questões, considerando todos os discentes, se dispôs para este trabalho do valor percentual de acertos nas questões validadas da prova. Já para a avaliação interna os dados empregados foram os referidos aos boletins de desempenho de 
todos os professores que ministraram as disciplinas envolvidas nas questões. Assim sendo, os dados são censitários.

O Componente de avaliação de Formação Geral do ENADE/2012 esteve composto por 10 (dez) questões, sendo 2 (duas) discursivas e 8 (oito) de múltipla escolha, abordando situações-problema, estudos de caso, simulações, interpretação de textos, imagens, gráficos e tabelas.

A parte relativa ao Componente de Conhecimento Específico da Área de Administração do ENADE/2012 foi elaborada atendendo à seguinte distribuição: 30 (trinta) questões, sendo 3 (três) discursivas com peso $15,0 \%$ e 27 (vinte e sete) de múltipla escolha com peso $85,0 \%$, envolvendo situações-problema e estudos de caso. Cabe assinalar que desse número foram anuladas seis. No Componente de Formação Geral, as 8 (oito) questões objetivas de múltipla escolha e as 2 (duas) discursivas tiveram pesos, respectivamente, iguais a $60,0 \%$ e $40,0 \%$.

No quesito da avaliação interna, para a análise, foram selecionados 38 professores das respectivas disciplinas envolvidas em cada questão. Após esta seleção, verificou-se em que período a disciplina foi ministrada e qual o professor que lecionou a mesma. Em seguida separou-se o boletim de desempenho do professor naquele ano em que a disciplina foi ministrada. Para este tipo de análise, o boletim foi diferenciado em suas 3 dimensões (DTC, HE e DPS) conforme o quadro 1, com os critérios avaliados.

Quadro 1 Critérios avaliados pela UNIVALI na Avaliação Docente.

\begin{tabular}{|l|c|l|}
\hline Dimensão & Critério & Nome do Critério \\
\hline Domínio Técnico & 1 & Tem domínio de conteúdo \\
Científico & 2 & Usa o plano de ensino como referência para o desenvolvimento da \\
(DTC), & 3 & disciplina \\
& 4 & Estabelece relações entre as disciplinas e a prática profissional \\
& 5 & O nível de complexidade das avaliações em relação ao conteúdo \\
& 6 & trabalhado \\
& & O nível de exigência da disciplina em relação ao perfil profissional \\
& O nível de importância para sua formação \\
\hline Habilidades de & 7 & Esclarece dúvidas \\
Ensino (HE) & 8 & Utiliza linguagem clara no desenvolvimento das estratégias de ensino \\
& 9 & Discute os resultados das avaliações com a turma \\
\hline Desenvolvimento & 10 & Mantém um clima de cooperação, respeito e trabalho produtivo na sala \\
Pessoal e & & de aula. \\
Situacional & & \\
(DPS) & & \\
\hline
\end{tabular}

Fonte: Adaptado da ProEn, 2012 
O trabalho foi realizado utilizando a base de dados da UNIVALI, da Gerência de Ensino e Avaliação (GEA) da Vice-Reitoria de Graduação. A base de dados utilizada foi a do processo de Avaliação de Desempenho Docente do curso de Administração do campus de Itajaí na percepção dos alunos. Esta avaliação objetiva promover um processo sistemático de geração de informações para o planejamento e a tomada de decisão com vistas à obtenção de padrões de excelência na ação universitária.

Os resultados da Avaliação Institucional ocorrem sempre em duas etapas:

a) Boletim individual de desempenho do professor. Este boletim está disponível na intranet através do menu Boletins Avaliação Institucional poucos dias após o término da pesquisa.

b) Relatórios por curso. Cada curso recebe um relatório disponibilizado aos gestores (diretores de centro, coordenadores de cursos e apoio pedagógico), em formato Word e utilizando o ambiente share point(https://extranet.univali.br/oficial/gea/default.aspx).

A coordenação de curso junto ao Núcleo Docente Estruturante - NDE e ao colegiado de curso analisam e discutem os resultados, propondo e realizando ações como: investimentos na formação continuada dos docentes, reuniões com professores, definição de critérios comuns para avaliação de aprendizagem, constantes atualizações das ementas, discussão conjunta dos projetos integrados, incentivo à realização de palestras com profissionais do mercado nas disciplinas, plano de ensino disponível na primeira semana de aula e análise das relações entre disciplinas e laboratórios.

\subsection{RELACIONAMENTO ENTRE A AVALIAÇÃO EXTERNA E INTERNA}

Para fazer a relação das avaliações externas e internas foram identificadas as disciplinas e os desempenhos segundo as questões em que os alunos participaram. Em seguida, foram mensuradas estas associações utilizando-se análise de correlação.

Os dados foram organizados numa planilha eletrônica para desenvolver as etapas descritas anteriormente. A seguir foram exportadas para um software estatístico com a finalidade de obter os coeficientes de associação e analisar as relações identificadas entre as avaliações.

A partir da avaliação externa, estavam disponíveis os resultados do desempenho nas questões das disciplinas nelas envolvidas. Da avaliação interna dos docentes pelos discentes, obteve-se uma medida para o desempenho do docente daquelas mesmas disciplinas, segundo os critérios já mencionados, isto é, o DTC, HE e DPS. 
Previamente à utilização da correlação paramétrica foram verificados os pressupostos para seu emprego. No caso do desempenho dos alunos e o domínio técnico científico (DTC) dos docentes verificou-se a normalidade dos dados. Contudo, para as dimensões habilidade de ensino (HE) e desenvolvimento pessoal e situacional (DPS) a mesma não se constatou, existindo a necessidade de empregar um método não paramétrico. Ou seja, se usou o coeficiente de correlação não paramétrico.

Os métodos paramétricos, segundo Martínez-Espinosa (2002), exigem que a(s) amostra(s) tenha(m) uma distribuição normal, especialmente se tiverem uma dimensão inferior a 30. Nas amostras de dimensão superior geralmente a distribuição aproxima-se da normalidade. Os métodos não paramétricos não necessitam de requisitos tão fortes como nos anteriores. E também são indicados quando as amostras são pequenas.

Com os valores do desempenho de alunos e o DTC se realizou a avaliação do relacionamento entre ambos os resultados pelo cálculo do coeficiente paramétrico de correlação de Pearson. Ele mede a força das associações que existem entre duas variáveis. É a covariação de variáveis padronizadas e, portanto, não há unidade de medida, variando de -1 até 1.

Para avaliar a correlação entre o desempenho discente com HE e com DPS foi empregado o coeficiente não paramétrico por postos ou ranking de Spearman. Tal qual o coeficiente de Pearson ele mede o vigor da relação entre as variáveis.

\section{RESULTADOS}

\subsection{ANÁLISE DESCRITIVA DAS VARIÁVEIS E RESULTADOS DO DESEMPENHO}

Nesta parte será apresentada uma análise descritiva das variáveis obtidas, abordando a importância de cada variável utilizada nos resultados da pesquisa.

\subsubsection{AVALIAÇÃO EXTERNA}

O desempenho acadêmico no ENADE foi medido por intermédio do relatório do curso, do qual foram analisados todos os conteúdos predominantes, identificando as disciplinas contempladas, o ano em que foi ministrada e a porcentagem de acertos da instituição. O resultado se exibe na Tabela 1 
Tabela 1 Conteúdos predominantes, disciplinas contempladas, período, ano e a porcentagem de acertos da instituição.

\begin{tabular}{|c|c|c|c|c|}
\hline Questão & Conteúdo & Disciplina & Ano & $\%$ \\
\hline $\begin{array}{l}01 \mathrm{FG} \\
\mathrm{ME}\end{array}$ & Políticas públicas (Educação) & $\begin{array}{c}\text { Antropologia Aplicada à } \\
\text { Gestão }\end{array}$ & 2009 & 63,8 \\
\hline $\begin{array}{c}02 \mathrm{FG} \\
\mathrm{ME}\end{array}$ & Ecologia/Biodiversidade & $\begin{array}{c}\text { Antropologia Aplicada à } \\
\text { Gestão }\end{array}$ & 2009 & 49,6 \\
\hline $\begin{array}{l}03 \mathrm{FG} \\
\mathrm{ME}\end{array}$ & $\begin{array}{l}\text { Políticas públicas } \\
\text { (Desenvolvimento } \\
\text { Sustentável) }\end{array}$ & $\begin{array}{l}\text { Antropologia Aplicada à } \\
\text { Gestão }\end{array}$ & 2009 & 50,9 \\
\hline $\begin{array}{l}04 \mathrm{FG} \\
\mathrm{ME}\end{array}$ & $\begin{array}{l}\text { Democracia, ética e } \\
\text { cidadania. }\end{array}$ & Filosofia e ética & 2010 & 48,7 \\
\hline $\begin{array}{l}05 \mathrm{FG} \\
\mathrm{ME}\end{array}$ & Globalização e geopolítica & Mercado Global & 2010 & 30,2 \\
\hline $\begin{array}{l}06 \mathrm{FG} \\
\mathrm{ME}\end{array}$ & Avanços tecnológicos & $\begin{array}{l}\text { Administração de Sistemas } \\
\text { de Informação }\end{array}$ & 2012 & 55,2 \\
\hline $\begin{array}{l}07 \mathrm{FG} \\
\mathrm{ME}\end{array}$ & Sociodiversidade & $\begin{array}{l}\text { Antropologia Aplicada à } \\
\text { Gestão }\end{array}$ & 2009 & 55,2 \\
\hline $\begin{array}{l}08 \mathrm{FG} \\
\mathrm{ME}\end{array}$ & Políticas públicas & $\begin{array}{l}\text { Antropologia Aplicada à } \\
\text { Gestão }\end{array}$ & 2009 & 45,7 \\
\hline $\begin{array}{l}01 \mathrm{FG} \\
\text { Dis }\end{array}$ & $\begin{array}{l}\text { Políticas públicas } \\
\text { (Desenvolvimento } \\
\text { sustentável) }\end{array}$ & $\begin{array}{c}\text { Antropologia Aplicada à } \\
\text { Gestão }\end{array}$ & 2009 & 32,9 \\
\hline \multirow{2}{*}{$\begin{array}{l}02 \text { FG } \\
\text { Dis }\end{array}$} & Violência & $\begin{array}{c}\text { Antropologia Aplicada à } \\
\text { Gestão }\end{array}$ & 2009 & 39,5 \\
\hline & Avanços tecnológicos & $\begin{array}{c}\text { Administração de Sistemas } \\
\text { de Informação }\end{array}$ & 2012 & 39,5 \\
\hline $\begin{array}{l}09 \mathrm{CE} \\
\mathrm{ME}\end{array}$ & Anulada & Anulada & Anulada & $\begin{array}{c}\text { Anul } \\
\text { ada }\end{array}$ \\
\hline $\begin{array}{l}10 \mathrm{CE} \\
\mathrm{ME}\end{array}$ & Anulada & Anulada & Anulada & $\begin{array}{c}\text { Anul } \\
\text { ada }\end{array}$ \\
\hline $\begin{array}{l}11 \mathrm{CE} \\
\mathrm{ME}\end{array}$ & Teorias da Administração & $\begin{array}{l}\text { Teorias da Administração I } \\
\text { Teorias da Administração II }\end{array}$ & $\begin{array}{l}2009 \\
2009\end{array}$ & 26,7 \\
\hline $\begin{array}{c}12 \mathrm{CE} \\
\mathrm{ME}\end{array}$ & Administração Financeira & $\begin{array}{l}\text { Administração Financeira I } \\
\text { Administração Financeira II }\end{array}$ & $\begin{array}{l}2010 \\
2011\end{array}$ & 56,0 \\
\hline \multirow{3}{*}{$\begin{array}{l}13 \mathrm{CE} \\
\mathrm{ME}\end{array}$} & Gestão de Custos & Gestão de Custos & 2010 & 38,8 \\
\hline & Administração Financeira & $\begin{array}{l}\text { Administração Financeira I } \\
\text { Administração Financeira II }\end{array}$ & $\begin{array}{l}2010 \\
2010\end{array}$ & 38,8 \\
\hline & Contabilidade Gerencial. & $\begin{array}{l}\text { Contabilidade Gerencial. } \\
\text { (eletiva) }\end{array}$ & 2010 & 38,8 \\
\hline $\begin{array}{c}14 \mathrm{CE} \\
\mathrm{ME}\end{array}$ & Administração Financeira & $\begin{array}{l}\text { Administração Financeira I } \\
\text { Administração Financeira II }\end{array}$ & $\begin{array}{l}2010 \\
2010\end{array}$ & 52,2 \\
\hline $\begin{array}{l}15 \mathrm{CE} \\
\mathrm{ME}\end{array}$ & $\begin{array}{c}\text { Responsabilidade Social e } \\
\text { Ambiental }\end{array}$ & $\begin{array}{c}\text { Responsabilidade Social e } \\
\text { Ambiental }\end{array}$ & 2010 & 54,7 \\
\hline $\begin{array}{l}16 \mathrm{CE} \\
\mathrm{ME}\end{array}$ & Estatística & Estatística & 2010 & 28,9 \\
\hline $\begin{array}{l}17 \mathrm{CE} \\
\mathrm{ME}\end{array}$ & Estatística & Estatística & 2010 & 31,9 \\
\hline $\begin{array}{l}18 \mathrm{CE} \\
\mathrm{ME}\end{array}$ & Estatística & Estatística & 2010 & 35,8 \\
\hline
\end{tabular}




\begin{tabular}{|c|c|c|c|c|}
\hline $\begin{array}{c}19 \mathrm{CE} \\
\mathrm{ME}\end{array}$ & $\begin{array}{c}\text { Administração da Produção e } \\
\text { Operações }\end{array}$ & $\begin{array}{l}\text { Administração da Produção e } \\
\text { Operações }\end{array}$ & 2011 & 43,5 \\
\hline $\begin{array}{l}20 \mathrm{CE} \\
\mathrm{ME}\end{array}$ & Administração Financeira & $\begin{array}{l}\text { Administração Financeira I } \\
\text { Administração Financeira II }\end{array}$ & $\begin{array}{l}2010 \\
2010\end{array}$ & 51,3 \\
\hline $\begin{array}{c}21 \mathrm{CE} \\
\mathrm{ME}\end{array}$ & Administração de Materiais & Administração de Materiais & 2010 & 29,7 \\
\hline $\begin{array}{c}22 \mathrm{CE} \\
\mathrm{ME} \\
\end{array}$ & Anulada & Anulada & Anulada & $\begin{array}{c}\text { Anul } \\
\text { ada }\end{array}$ \\
\hline $\begin{array}{l}23 \mathrm{CE} \\
\mathrm{ME}\end{array}$ & $\begin{array}{l}\text { Administração de Sistema de } \\
\text { Informação }\end{array}$ & $\begin{array}{c}\text { Administração de Sistemas } \\
\text { de Informação }\end{array}$ & 2012 & 23,7 \\
\hline $\begin{array}{l}24 \mathrm{CE} \\
\mathrm{ME}\end{array}$ & Ética & Filosofia e ética & 2010 & 41,8 \\
\hline $\begin{array}{c}25 \mathrm{CE} \\
\mathrm{ME}\end{array}$ & Anulada & Anulada & Anulada & $\begin{array}{c}\text { Anul } \\
\text { ada }\end{array}$ \\
\hline $\begin{array}{l}26 \mathrm{CE} \\
\mathrm{ME}\end{array}$ & Anulada & Anulada & Anulada & $\begin{array}{c}\text { Anul } \\
\text { ada }\end{array}$ \\
\hline $\begin{array}{l}27 \mathrm{CE} \\
\mathrm{ME}\end{array}$ & $\begin{array}{c}\text { Administração de Recursos } \\
\text { Humanos }\end{array}$ & $\begin{array}{l}\text { Administração de Recursos } \\
\text { Humanos I } \\
\text { Administração de Recursos } \\
\text { Humanos II } \\
\end{array}$ & $\begin{array}{l}2011 \\
2011\end{array}$ & 31,0 \\
\hline $\begin{array}{c}28 \mathrm{CE} \\
\mathrm{ME}\end{array}$ & Estatística & Estatística & 2010 & 40,5 \\
\hline $\begin{array}{l}29 \mathrm{CE} \\
\mathrm{ME}\end{array}$ & Anulada & Anulada & Anulada & $\begin{array}{c}\text { Anul } \\
\text { ada }\end{array}$ \\
\hline $\begin{array}{l}30 \mathrm{CE} \\
\mathrm{ME}\end{array}$ & $\begin{array}{l}\text { Administração } \\
\text { Mercadológica }\end{array}$ & $\begin{array}{l}\text { Administração } \\
\text { Mercadológica I } \\
\text { Administração } \\
\text { Mercadológica II }\end{array}$ & $\begin{array}{l}2011 \\
2011\end{array}$ & 57,3 \\
\hline $\begin{array}{c}31 \mathrm{CE} \\
\mathrm{ME} \\
\end{array}$ & $\begin{array}{c}\text { Responsabilidade Social e } \\
\text { Ambiental } \\
\end{array}$ & $\begin{array}{c}\text { Responsabilidade Social e } \\
\text { Ambiental }\end{array}$ & 2010 & 28,4 \\
\hline $\begin{array}{c}32 \mathrm{CE} \\
\mathrm{ME}\end{array}$ & Estratégias Organizacionais & Estratégias Organizacionais & 2012 & 37,1 \\
\hline $\begin{array}{c}33 \mathrm{CE} \\
\mathrm{ME}\end{array}$ & Estratégias Organizacionais & Estratégias Organizacionais & 2012 & 42,7 \\
\hline $\begin{array}{l}34 \mathrm{CE} \\
\mathrm{ME}\end{array}$ & Estratégias Organizacionais & Estratégias Organizacionais & 2012 & 35,3 \\
\hline $\begin{array}{l}35 \mathrm{CE} \\
\mathrm{ME}\end{array}$ & $\begin{array}{c}\text { Administração de Recursos } \\
\text { Humanos }\end{array}$ & $\begin{array}{c}\text { Administração de Recursos } \\
\text { Humanos I } \\
\text { Administração de Recursos } \\
\text { Humanos II } \\
\end{array}$ & $\begin{array}{l}2011 \\
2011\end{array}$ & 53,4 \\
\hline \multirow{3}{*}{$\begin{array}{l}03 \mathrm{CE} \\
\text { Dis }\end{array}$} & Estatística, & Estatística & 2010 & 11,0 \\
\hline & $\begin{array}{l}\text { Administração } \\
\text { Mercadológica }\end{array}$ & $\begin{array}{l}\text { Administração } \\
\text { Mercadológica I } \\
\text { Administração } \\
\text { Mercadológica II } \\
\end{array}$ & $\begin{array}{l}2011 \\
2011\end{array}$ & 11,0 \\
\hline & $\begin{array}{l}\text { Análise Financeira de } \\
\text { Investimentos. }\end{array}$ & $\begin{array}{l}\text { Avaliação Financeira de } \\
\text { Investimentos }\end{array}$ & 2009 & 11,0 \\
\hline \multirow{3}{*}{$\begin{array}{l}04 \mathrm{CE} \\
\text { Dis }\end{array}$} & $\begin{array}{l}\text { Administração de Sistema de } \\
\text { Informação }\end{array}$ & $\begin{array}{c}\text { Administração de Sistemas } \\
\text { de Informação }\end{array}$ & 2012 & 7,6 \\
\hline & Inovação e Criatividade & Inovação e Criatividade & 2010 & 7,6 \\
\hline & Mercado de Capitais & Mercado de Capitais & 2011 & 7,6 \\
\hline
\end{tabular}




\begin{tabular}{c|c|c|c|c}
\hline & $\begin{array}{c}\text { Organização de Sistemas e } \\
\text { Métodos. }\end{array}$ & $\begin{array}{c}\text { Organização de Sistemas e } \\
\text { Métodos }\end{array}$ & 2010 & 7,6 \\
\hline \multirow{4}{*}{$\begin{array}{c}\text { 05 CE } \\
\text { Dis }\end{array}$} & $\begin{array}{c}\text { Administração de Recursos } \\
\text { Humanos, }\end{array}$ & $\begin{array}{c}\text { Administração de Recursos } \\
\text { Humanos I } \\
\text { Administração de Recursos } \\
\text { Humanos II }\end{array}$ & $\begin{array}{c}2011 \\
2011\end{array}$ & 25,4 \\
\cline { 2 - 5 } & $\begin{array}{c}\text { Administração da Produção e } \\
\text { Operações, }\end{array}$ & $\begin{array}{c}\text { Administração da Produção e } \\
\text { Operações }\end{array}$ & 2011 & 25,4 \\
\cline { 2 - 5 } & Projetos Organizacionais & Projetos organizacionais & 2012 & 25,4 \\
\cline { 2 - 5 } & Administração & $\begin{array}{c}\text { Administração } \\
\text { Mercadológica I } \\
\text { Administração } \\
\text { mercadológica II }\end{array}$ & 2011 & 25,4 \\
& Mercadológica. & 2011 & 25,4 \\
\hline
\end{tabular}

Fonte dos dados: INEP (2014)

FG ME - Formação geral múltipla escolha

FG Dis - Formação geral discursiva

CE ME - Conhecimento específico múltipla escolha

CE Dis - Conhecimento específico discursiva

\subsubsection{AVALIAÇÃO INTERNA}

A avaliação do corpo docente realizada pelos alunos foi medida por meio do boletim de desempenho individual do professor, que inclui três dimensões. Avalia-se o Domínio Técnico Científico (DTC), Habilidades de Ensino (HE) e Desenvolvimento Pessoal e Situacional (DPS), calculando-se a média das notas atribuídas por todos os discentes nos critérios analisados.

Em seguida foram geradas as Tabelas 2, 3, 4 e 5, correspondentes aos componentes e modalidades de resposta, onde se ordenaram do mais alto para o mais baixo desempenho nas questões das disciplinas envolvidas. Relacionaram-se conjuntamente os desempenhos dos docentes segundo as três dimensões: DTC, HE e DPS.

Tabela 2 Questões do componente específico - múltipla escolha

\begin{tabular}{c|l|c|c|c|c}
\hline Questão & \multicolumn{1}{|c|}{ Disciplina } & Desempenho & $\begin{array}{c}\text { Dimensão } \\
\text { DTC }\end{array}$ & $\begin{array}{c}\text { Dimensão } \\
\text { HE }\end{array}$ & $\begin{array}{c}\text { Dimensão } \\
\text { DPS }\end{array}$ \\
\hline 30 & Administração Mercadológica I & $57,3 \%$ & 8,51 & 9,14 & 9,60 \\
\hline 30 & Administração Mercadológica II & $57,3 \%$ & 7,49 & 7,22 & 7,98 \\
\hline 12 & Administração Financeira I & $56,0 \%$ & 7,78 & 7,80 & 8,98 \\
\hline 12 & Administração Financeira II & $56,0 \%$ & 7,58 & 7,22 & 7,74 \\
\hline 15 & $\begin{array}{l}\text { Responsabilidade Social e } \\
\text { Ambiental }\end{array}$ & $54,7 \%$ & 8,16 & 8,70 & 9,04 \\
\hline 35 & $\begin{array}{l}\text { Administração de Recursos } \\
\text { Humanos I }\end{array}$ & $53,4 \%$ & 8,48 & 8,76 & 9,14 \\
\hline 35 & $\begin{array}{l}\text { Administração } \\
\text { Humanos II }\end{array}$ & $53,4 \%$ & 8,56 & 8,20 & 8,84 \\
\hline 14 & Administração Financeira I & $52,2 \%$ & 7,78 & 7,80 & 8,98 \\
\hline
\end{tabular}




\begin{tabular}{|c|c|c|c|c|c|}
\hline 14 & Administração Financeira II & $52,2 \%$ & 7,58 & 7,22 & 7,74 \\
\hline 20 & Administração Financeira I & $51,3 \%$ & 7,78 & 7,80 & 8,98 \\
\hline 20 & Administração Financeira II & $51,3 \%$ & 7,58 & 7,22 & 7,74 \\
\hline 19 & $\begin{array}{l}\text { Administração da Produção e } \\
\text { Operação }\end{array}$ & $43,5 \%$ & 7,42 & 7,20 & 7,99 \\
\hline 33 & Estratégias Organizacionais & $42,7 \%$ & 7,38 & 6,89 & 7,68 \\
\hline 24 & Filosofia e Ética & $41,8 \%$ & 7,69 & 7,49 & 7,52 \\
\hline 28 & Estatística & $40,5 \%$ & 8,11 & 8,87 & 9,06 \\
\hline 13 & Administração Financeira I & $38,8 \%$ & 7,78 & 7,80 & 8,98 \\
\hline 13 & Administração Financeira II & $38,8 \%$ & 7,58 & 7,22 & 7,74 \\
\hline 13 & Gestão de Custos & $38,8 \%$ & 8,27 & 8,51 & 8,76 \\
\hline 13 & Contabilidade Gerencial & $38,8 \%$ & 7,46 & 7,04 & 7,48 \\
\hline 32 & Estratégias Organizacionais & $37,1 \%$ & 7,38 & 6,89 & 7,68 \\
\hline 18 & Estatística & $35,8 \%$ & 8,11 & 8,87 & 9,06 \\
\hline 34 & Estratégias Organizacionais & $35,3 \%$ & 7,38 & 6,89 & 7,68 \\
\hline 17 & Estatística & $31,9 \%$ & 8,11 & 8,87 & 9,06 \\
\hline 27 & $\begin{array}{l}\text { Administração de } \\
\text { Humanos I }\end{array}$ & $31,0 \%$ & 8,48 & 8,76 & 9,14 \\
\hline 27 & $\begin{array}{ll}\text { Administração } & \text { Recursos } \\
\text { Humanos II } & \end{array}$ & $31,0 \%$ & 8,56 & 8,20 & 8,84 \\
\hline 21 & Administração de Materiais & $29,7 \%$ & 8,02 & 8,34 & 8,41 \\
\hline 16 & Estatística & $28,9 \%$ & 8,11 & 8,87 & 9,06 \\
\hline 31 & $\begin{array}{lll}\text { Responsabilidade } & \text { Social } & \mathrm{e} \\
\text { Ambiental } & & \\
\end{array}$ & $28,4 \%$ & 8,16 & 8,70 & 9,04 \\
\hline 11 & Teoria da Administração I & $26,7 \%$ & 8,35 & 8,03 & 8,18 \\
\hline 11 & Teoria da Administração II & $26,7 \%$ & 7,97 & 7,60 & 8,21 \\
\hline 23 & $\begin{array}{l}\text { Administração de Sistemas de } \\
\text { Informação }\end{array}$ & $23,7 \%$ & 6,75 & 7,09 & 7,61 \\
\hline
\end{tabular}

Fonte: ProEn 2014

Fonte dos dados: INEP (2014)

Tabela 3 Questões componente específico - discursiva

\begin{tabular}{c|l|c|c|c|c}
\hline Questão & \multicolumn{1}{|c|}{ Disciplina } & Desempenho & $\begin{array}{c}\text { Dimensão } \\
\text { DTC }\end{array}$ & $\begin{array}{c}\text { Dimensão } \\
\text { HE }\end{array}$ & $\begin{array}{c}\text { Dimensão } \\
\text { DPS }\end{array}$ \\
\hline 05 & Administração Mercadológica I & $25,4 \%$ & 8,51 & 9,14 & 9,60 \\
\hline 05 & Administracão Mercadológica II & $25,4 \%$ & 7,49 & 7,22 & 7,98 \\
\hline 05 & $\begin{array}{l}\text { Administração de Recursos } \\
\text { Humanos I }\end{array}$ & $25,4 \%$ & 8,48 & 8,76 & 9,14 \\
\hline 05 & $\begin{array}{l}\text { Administração Recursos Humanos } \\
\text { II }\end{array}$ & $25,4 \%$ & 8,56 & 8,20 & 8,84 \\
\hline 05 & $\begin{array}{l}\text { Administração da Produção e } \\
\text { Operaç }\end{array}$ & $25,4 \%$ & 7,42 & 7,20 & 7,99 \\
\hline 05 & Projetos Organizacionais & $25,4 \%$ & 8,79 & 9,23 & 9,48 \\
\hline 03 & Estatística & $11,0 \%$ & 8,11 & 8,87 & 9,06 \\
\hline 03 & $\begin{array}{l}\text { Avaliação } \\
\text { Investimentos }\end{array}$ & $11,0 \%$ & 8,85 & 8,84 & 8,79 \\
\hline 03 & Administração Mercadológica I & $11,0 \%$ & 8,51 & 9,14 & 9,60 \\
\hline 03 & Administração Mercadológica II & $11,0 \%$ & 7,49 & 7,22 & 7,98 \\
\hline 04 & Organização, Sistemas e Métodos & $7,6 \%$ & 6,96 & 7,08 & 7,57 \\
\hline 04 & Mercado de Capitais & $7,6 \%$ & 7,72 & 7,39 & 8,49 \\
\hline
\end{tabular}


Fonte: ProEn 2014

Fonte dos dados: INEP (2014)

Tabela 4 Questões de formação geral - múltipla escolha

\begin{tabular}{c|l|c|c|c|c}
\hline Questão & \multicolumn{1}{|c|}{ Disciplina } & Desempenho & $\begin{array}{c}\text { Dimensão } \\
\text { DTC }\end{array}$ & $\begin{array}{c}\text { Dimensão } \\
\text { HE }\end{array}$ & $\begin{array}{c}\text { Dimensão } \\
\text { DPS }\end{array}$ \\
\hline 01 & Antropologia Aplicada a Gestão & $63,8 \%$ & 7,91 & 8,34 & 8,57 \\
\hline 06 & $\begin{array}{l}\text { Administração de Sistemas de } \\
\text { Informação }\end{array}$ & $55,2 \%$ & 6,75 & 7,09 & 7,61 \\
\hline 07 & Antropologia Aplicada a Gestão & $55,2 \%$ & 7,91 & 8,34 & 8,57 \\
\hline 03 & Antropologia Aplicada a Gestão & $50,9 \%$ & 7,91 & 8,34 & 8,57 \\
\hline 02 & Antropologia Aplicada a Gestão & $49,6 \%$ & 7,91 & 8,34 & 8,57 \\
\hline 04 & Filosofia e Ética & $48,7 \%$ & 7,69 & 7,49 & 7,52 \\
\hline 08 & Antropologia Aplicada a Gestão & $45,7 \%$ & 7,91 & 8,34 & 8,57 \\
\hline 05 & Mercado Global & $30,2 \%$ & 8,86 & 9,35 & 9,32 \\
\hline
\end{tabular}

Fonte: ProEn 2014

Fonte dos dados: INEP (2014)

Tabela 5 Questões de formação geral - discursiva

\begin{tabular}{c|l|c|c|c|c}
\hline Questão & \multicolumn{1}{|c|}{ Disciplina } & Desempenho & $\begin{array}{c}\text { Dimensão } \\
\text { DTC }\end{array}$ & $\begin{array}{c}\text { Dimensão } \\
\text { HE }\end{array}$ & $\begin{array}{c}\text { Dimensão } \\
\text { DPS }\end{array}$ \\
\hline 02 & Antropologia Aplicada a Gestão & $39,5 \%$ & 7,91 & 8,34 & 8,57 \\
\hline 02 & $\begin{array}{l}\text { Administração de Sistemas de } \\
\text { Informação }\end{array}$ & $39,5 \%$ & 6,75 & 7,09 & 7,61 \\
\hline 01 & Antropologia Aplicada a Gestão & $32,9 \%$ & 7,91 & 8,34 & 8,57 \\
\hline
\end{tabular}

Fonte: ProEn 2014

Fonte dos dados: INEP (2014)

No item seguinte se expõem os resultados das associações analisadas.

\subsection{ANÁLISE DO RELACINAMENTO ENTRE A AVALIAÇÃO EXTERNA E INTERNA}

Com a finalidade de poder definir que coeficiente empregar para analisar o relacionamento entre a avaliação externa e interna fez-se inicialmente um teste de normalidade com os dados de desempenho discente em todas as questões do ENADE e do desempenho docente das disciplinas envolvidas considerando as três dimensões. Para tanto foi usado o teste de Shapiro-Wilk, que é adequado para pequenas amostras.

Os resultados obtidos indicam que o desempenho dos alunos e a dimensão domínio técnico científico (DTC) são normais ( $\mathrm{p}>\alpha$ ), e que as dimensões habilidade de ensino (HE) e desempenho pessoal e situacional (DPS) não tem distribuições normais $(p<\alpha)$. Em tal circunstância as relações foram mensuradas com o coeficiente paramétrico de Pearson no caso 
do desempenho dos estudantes com a dimensão DTC e com o coeficiente não paramétrico de Spearman na avaliação da associação do desempenho no ENADE com HE e com DPS.

Previamente ao calcular o coeficiente foi feito um diagrama de espalhamento com os diagramas Box-Plots para as variáveis que se estavam representando. Na Figura 1 se exibe a relação entre o desempenho dos alunos na abscissa e o domínio técnico científico na ordenada.

Figura 1 Diagrama de dispersão entre o desempenho discente e o domínio técnico científico (DTC). Na parte superior e no lateral direito encontram-se os box-plots daquelas variáveis.

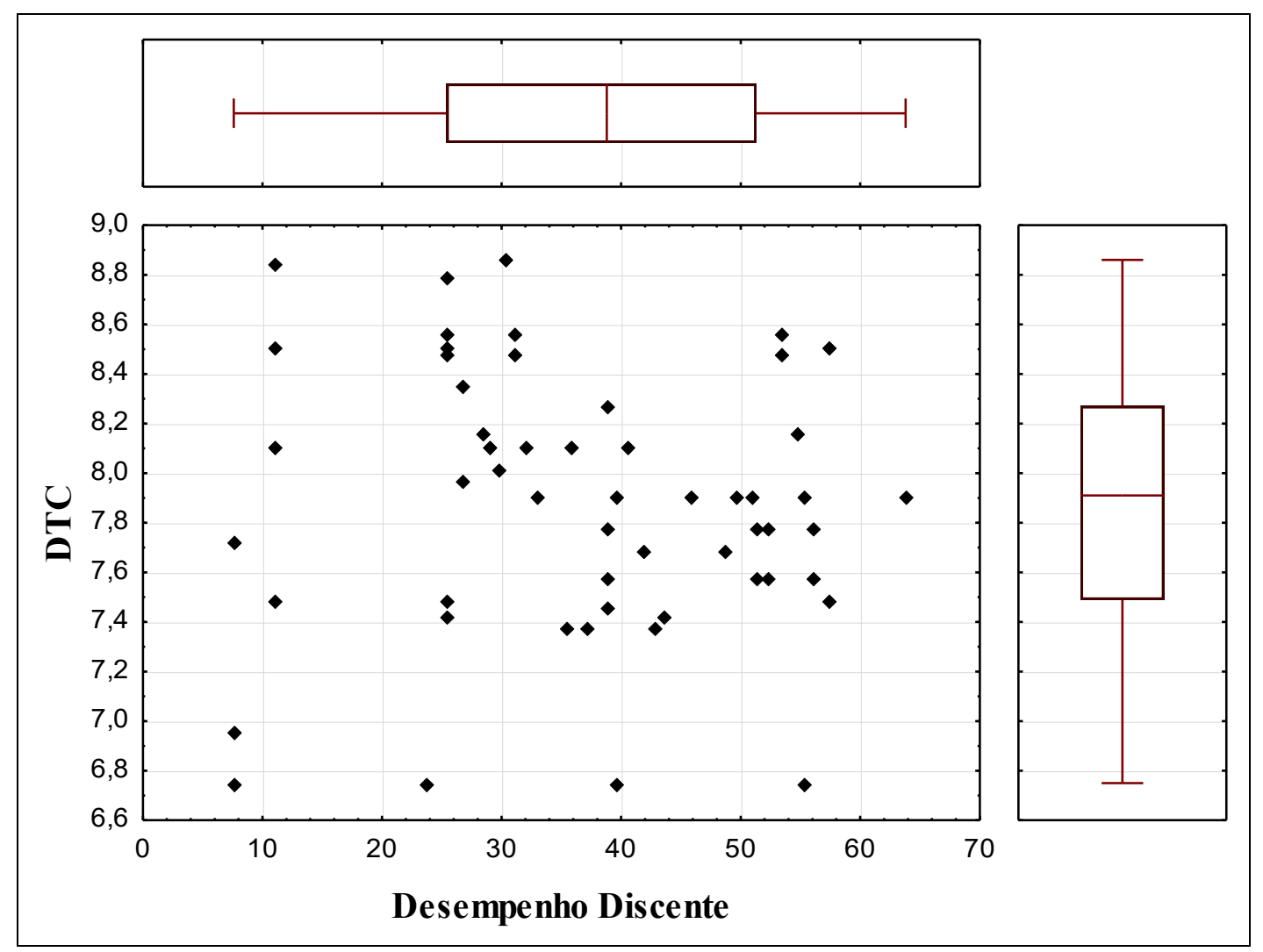

Fonte: Dados da pesquisa.

Observando a representação gráfica dos box-plots percebe-se que as variáveis são normais, mostrando simetria entre os setores das caixas e dos extremos. Por sua vez, o diagrama de dispersão mostra um espalhamento bastante aleatório, sem mostrar alguma associação observável. Isto é, com valores pequenos de desempenho se vinculam valores altos e baixos de DTC e vice-versa com valores altos há tanto desempenhos técnicos científicos altos quanto baixos.

Essa configuração se expressa num coeficiente de correlação de Pearson pequeno e não significativo $(\mathrm{p}=0,413)$, de apenas $-0,1350$. Contudo é interessante assinalar que o 
sentido do relacionamento é negativo, pois isso significa que a tendência é que os valores baixos de desempenho estudantil se encontram mais relacionado com valores altos de DTC e vice-versa, ou seja, os alunos que tiveram desempenho baixo no ENADE avaliaram bem o professor. Isto é mais evidente quando se analisa o desempenho e o DTC das questões de formação geral e múltipla escolha. Nesse caso o coeficiente de Pearson atinge -0,6360 e uma significância de $10 \%(\mathrm{p}=0,09)$,

Nas Figuras 2 e 3 se mostram os diagramas de dispersão e os box-plots entre os desempenhos no ENADE e a habilidade de ensino (HE) e o desenvolvimento pessoal e situacional (DPS) respectivamente. Em ambas as figuras podem ser observadas que aquelas dimensões do desempenho docente não possuem distribuição normal, pois os box-plots aparecem com notória assimetria.

Figura 2 Diagrama de dispersão entre o desempenho discente e a habilidade de ensino (HE). Na parte superior e no lateral direito encontram-se os box-plots daquelas variáveis.

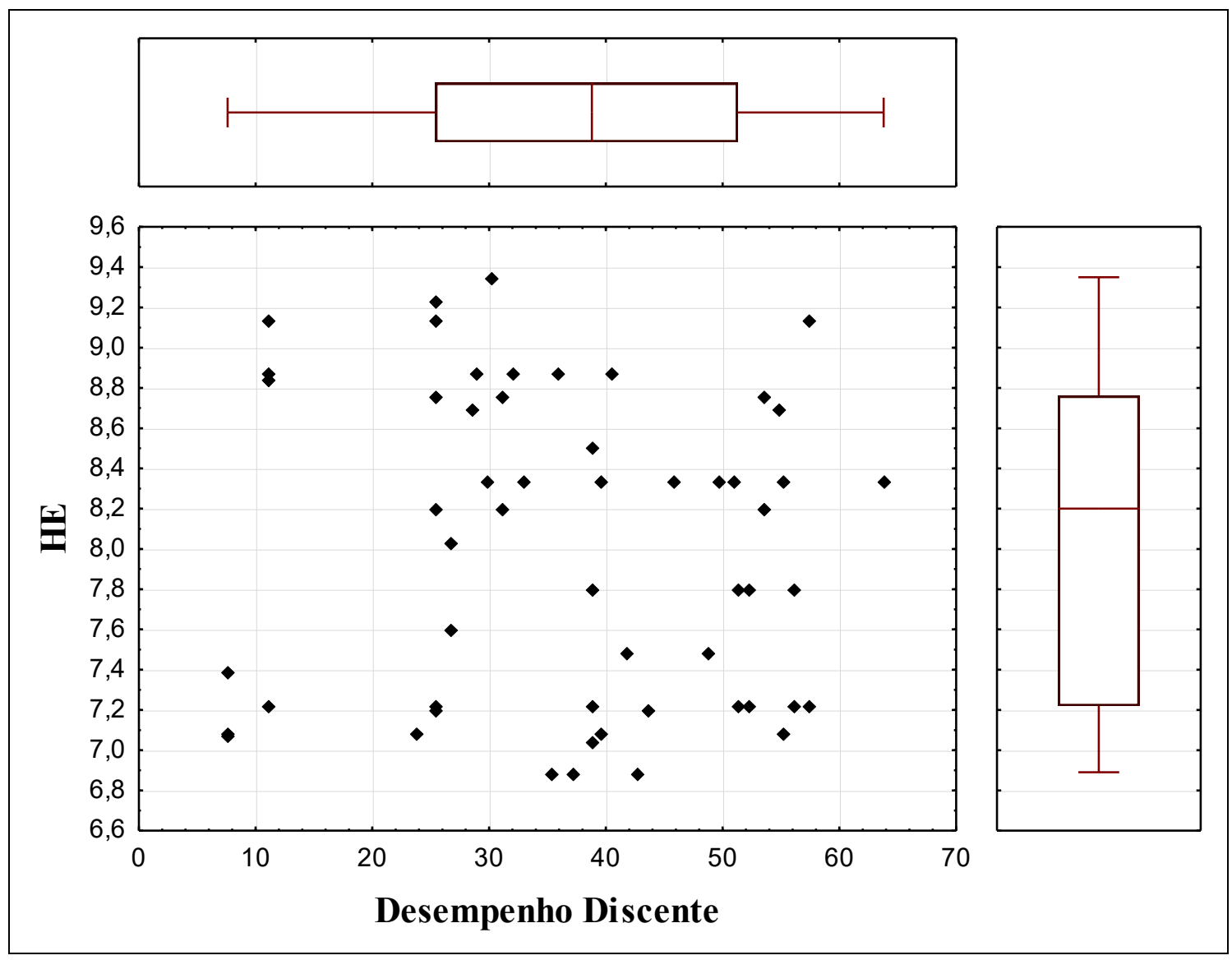

Fonte: Dados da pesquisa.

De igual maneira ao exposto na Figura 1, para o domínio técnico científico, também se verifica nos diagramas destas outras dimensões que não há padrões reconhecíveis na 
distribuição dos pontos que representam as variáveis em analise. Ou seja, os valores baixos do desempenho dos alunos podem mostrar valores altos ou baixos da dimensão do desempenho docente que se esteja considerando. Também pode ser observado que as mesmas condições se constatam para as dimensões HE e DPS confrontadas com o desempenho dos alunos.

Na Figura 3 se apresenta o diagrama de dispersão para a dimensão desenvolvimento pessoal e situacional em relação com o desempenho discente no ENADE. Nela podem ser verificadas as colocações feitas anteriormente.

Figura 3 Diagrama de dispersão entre o desempenho discente e o desenvolvimento pessoal e situacional (DPS). Na parte superior e no lateral direito encontram-se os box-plots daquelas variáveis.

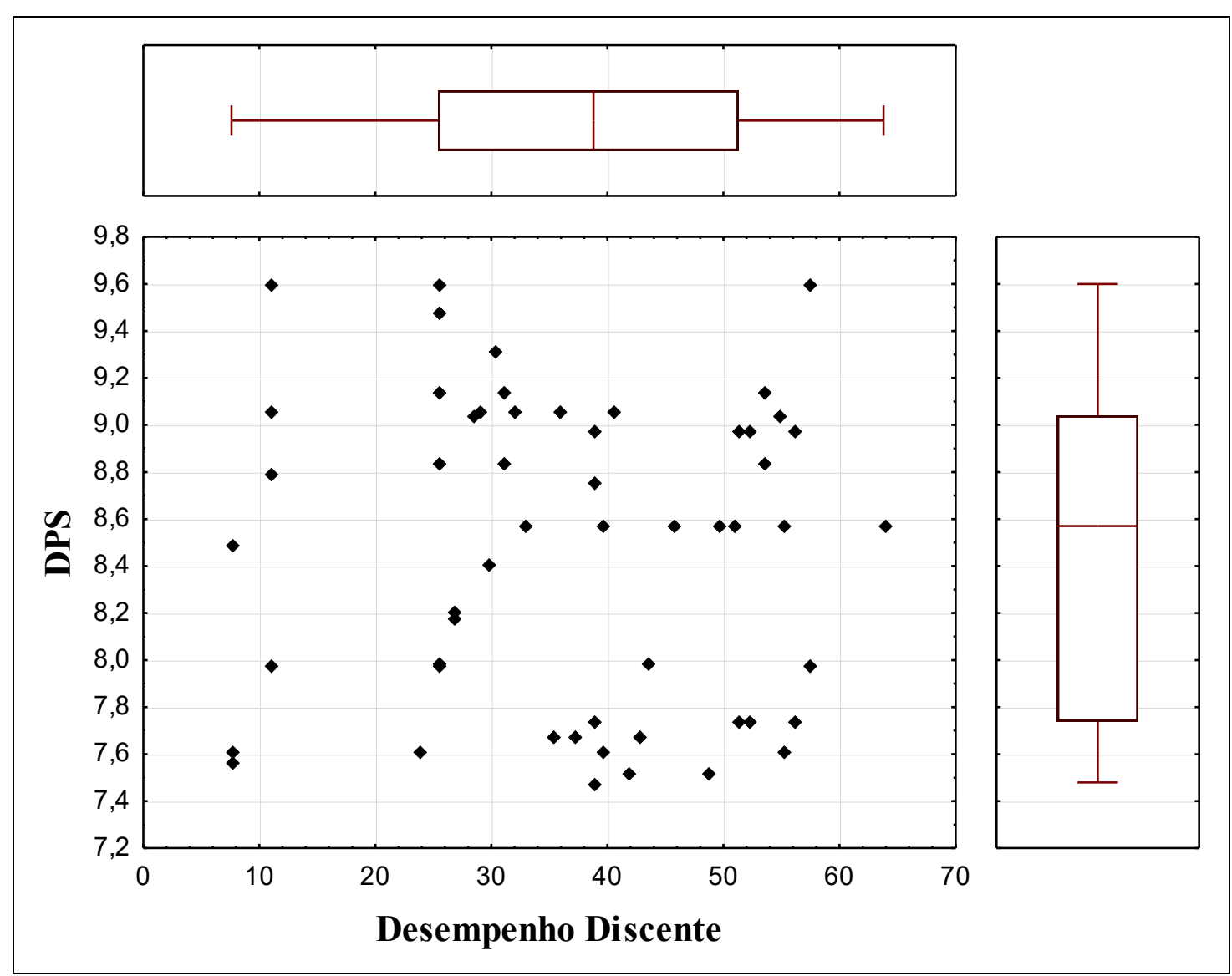

Fonte: Dados da pesquisa.

Considerando a falta de normalidade das distribuições dos valores de HE e DPS os coeficientes de correlação calculados para mensurar as relações com o desempenho dos alunos foram o de Spearman. A intensidade da relação se calcula usando, ao invés dos valores observados, os postos que ocupam os mesmos segundo o ordenamento de suas magnitudes.

Os coeficientes obtidos foram praticamente nulos, com valores de $-0,07$ e $-0,06$ respectivamente para as associações entre o desempenho discente com HE e com DPS. 
Portanto, também se confirma que não existe relação entre estas dimensões e o desempenho da instituição no ENADE.

\section{CONSIDERAÇÕES FINAIS}

Este artigo procurou responder à questão: Qual a relação entre os desempenhos docente e discente nos processos de avaliação interna e externa do Curso de Administração da UNIVALI de Itajaí?

A pesquisa iniciou-se em fontes de referências bibliográficas de relevância na academia, utilizando-se de artigos nacionais e estrangeiros, para que se conhecesse a Avaliação da Educação Superior em nível de Brasil e Internacional, dando destaque mais para o Brasil com relação ao ENADE e também à Avaliação Institucional na UNIVALI.

O objetivo geral foi atingido a partir da análise feita entre desempenho docente $\mathrm{e}$ discente, tendo como referencial o resultado da prova do ENADE 2012. O desempenho do estudante está embasado no relatório do curso publicado pelo INEP/MEC; já o do professor, nos boletins institucionais de desempenho dos ministrantes das disciplinas cujos conteúdos são contemplados nas questões da prova.

Dessa forma, este trabalho deu início às discussões da relação entre o desempenho docente e discente nos processos de avaliação interna e externa do Curso de Administração da UNIVALI de Itajaí.

Os dados revelados contribuem significativamente para a avaliação formativa. Uma vez que oferecem subsídios para a implementação de programas que alimentem o compromisso com a melhoria do ensino e assegurem a qualidade, tanto no nível da instituição como no do sistema.

Em relação a tabela 1 percebeu-se que o conteúdo mais contemplado foi o de Políticas Públicas, nas questões de Formação Geral, envolvendo a disciplina de Antropologia Aplicada à Gestão, ministrada no $1^{\circ}$ período do curso.

Já nas questões de Conhecimento Específico, a disciplina de Estatística, foi a mais cobrada pelo ENADE, seguida de Administração Financeira, Administração de Recursos Humanos, e Estratégias Organizacionais.

Quanto às avaliações do desempenho dos docentes que ministraram as disciplinas envolvidas nas questões do ENADE consideraram-se as dimensões do Domínio Técnico Científico (DTC), das Habilidades de Ensino (HE) e do Desenvolvimento Pessoal e Situacional (DPS). 
Neste item, o melhor DTC é o da disciplina Mercado Global, com 8,86, tendo como conteúdo tema de Formação Geral, com um índice de acerto na prova de 30,2\%, correspondendo à questão de $\mathrm{n}^{\mathrm{o}} 5$. (Tabela 4)

A disciplina Avaliação Financeira de Investimento também teve um bom desempenho quanto ao DTC, com 8,85, mas em contrapartida o acerto dos alunos na prova foi de apenas $11 \%$ e a questão analisada é a de $n^{0} 3$, de Conhecimento Específico. (Tabela 3). Observou-se aqui que o DTC foi alto enquanto que o percentual de acerto foi muito baixo, o que significa dizer que o professor não sabe passar o conteúdo? Será que a disciplina é de difícil entendimento?

Já a disciplina Administração de Sistemas de Informação, teve um DTC de 6,75 e constam nas questões de $\mathrm{n}^{\mathrm{o}} 6$ de Formação Geral, com índice de 55,2\% de acerto dos alunos; (tabela 4), na questão 2, também de Formação Geral, com 39,5\%, (tabela 5), na questão 23, de Componente Específico, com $23,7 \%$ de acerto (tabela 2) e na questão 4, também de Componente Específico, com apenas 7,6\% de acerto dos alunos. (Tabela 3)

Outra disciplina que também teve um baixo desempenho no DTC foi Organização de Sistemas e Métodos, com 6,96. Esta compôs a questão de $n^{\circ} 4$ de Componente Específico e nossos alunos acertaram apenas 7,6\%. (Tabela 3)

Quanto ao HE, o melhor desempenho foi de 9,35, cuja disciplina é a de Mercado Global e fez parte das questões de Formação Geral, foi a $5^{\text {a }}$ questão da prova do ENADE, com um acerto de 30,2\%. (Tabela 4). Quase o mesmo desempenho verificou-se na disciplina Projetos Organizacionais, com 9,23 de desempenho, também foi a $5^{\text {a }}$ questão da prova de Componente Específico, com desempenho de 25,4\%. (Tabela 3).

Ainda sobre HE, o pior desempenho foi na disciplina Estratégias Organizacional, com apenas 6,89. Esta disciplina fez parte da questão do ENADE de $\mathrm{n}^{\mathbf{0}} 32 \mathrm{em}$ que os alunos acertaram 37,1\%; questão $n^{\circ} 33$, com acerto de $42,7 \%$ e da questão $n^{\circ} 34$, com $35,3 \%$ de desempenho dos alunos. (Tabela 2). Essas questões fazem parte do Componente Específico.

Em se tratando de DPS, o melhor desempenho para o professor foi de 9,60 na disciplina Administração Mercadológica I, fazendo parte do ENADE nas questões de número 30 , (tabela 2) com acerto de 57,3\%; $n^{\circ} 3$, com um percentual de acerto de $11 \%$ e da questão $\mathrm{n}^{\mathrm{0}} 5$, com desempenho de 25,4. (Tabela 3). Essas questões também fizeram parte do Componente Específico de estudo. 
Outro bom desempenho foi na questão de $\mathrm{n}^{0} 5$, cuja disciplina é Projetos Organizacionais, com 9,48 de desempenho do professor, enquanto que os alunos tiveram um acerto de 25,4\%, também fazendo parte dos componentes específicos. (Tabela 3 )

O desempenho mais baixo que tivemos em DPS foi de 7,48 na disciplina Contabilidade Gerencial, cuja questão foi a de $n^{0} 13$ com um percentual de acerto dos alunos de $38,8 \%$, fazendo parte do componente específico. (Tabela 2)

Em que se trata de relacionar o desempenho por questões da prova com o desempenho dos docentes que ministraram as disciplinas envolvidas nessas questões chegou-se ao resultado conforme expresso no diagrama de dispersão: na figura 1 se obtém um espalhamento bastante aleatório, não mostrando associação observável entre DTC e desempenho discente, observando ainda que o sentido do relacionamento é negativo, significando que a tendência é de que os valores baixos de desempenho discente se encontrem mais relacionados com valores altos de DTC e vice-versa, principalmente nas questões de formação geral e múltipla escolha.

Quanto à dimensão HE e DPS (figuras 2 e 3), também se verifica a inexistência de padrões reconhecíveis na distribuição dos pontos que representam as variáveis em análise. Significa dizer que aos valores baixos do desempenho dos alunos podem corresponder valores altos ou baixos da dimensão do desempenho docente.

Observa-se, portanto, que os coeficientes obtidos foram praticamente nulos, ou seja, confirma-se que não existe relação entre estas dimensões (HE e DPS) e o desempenho da Instituição no ENADE.

Neste sentido, fica evidente que por meio dos resultados obtidos na pesquisa não se pode afirmar que haja coerência entre a performance acadêmica na avaliação externa e a avaliação interna feita pelos discentes quanto ao desempenho dos professores das disciplinas envolvidas nas questões.

Portanto, chega-se à conclusão pelas análises feitas que a Avaliação Externa (ENADE) e a Avaliação Interna (Docentes) não são complementares, ou seja, um ótimo (ou um insatisfatório) desempenho nas questões do ENADE, não significa necessariamente uma Instituição com um ótimo (ou insatisfatório) desempenho docente na visão do corpo discente.

\section{REFERÊNCIAS}

BABBIE, E. Métodos de Pesquisas de Survey. Belo Horizonte: Editora UFMG, 2001, 519 p 
BLACKBURN, R.; PUTTEN, J. V. PENNA FIRME, T. Avaliação de docentes e do ensino. Universidade de Brasília/Cátedra UNESCO de Educação a Distância, Brasília, 1998.

BOYER, E.L. Scholarship reconsidered: Priorities of the professoriate. Princeton, NJ: Carnegie Foundation for the Advancement of Teaching. 1990.

BOWEN, H.R. \& SCHUSTER, J.H. American professors: A national resource imperiled. New York: Oxford University Press. 1986

BRASIL.MEC. Lei n. 10.861, de 14 de abril de 2004b. Institui o Sistema Nacional de Avaliação da educação Superior - SINAES e dá outras providências. Disponível em http://www.planalto.gov.br/ccivil_03/_ato2004-2006/2004/lei/110.861.htm. Acesso em: 27 abr.2014.

FONSECA, J. J. S. Metodologia da pesquisa científica. Fortaleza: UEC, 2002. Apostila.

INSTITUTO NACIONAL DE ESTUDOS E PESQUISAS EDUCACIONAIS ANÍSIO TEIXEIRA - INEP. ENADE 2012: perguntas frequentes. Disponível em:

$<$ http://portal.inep.gov.br/perguntas-frequentes1>. Acesso em: 26 abr. 2014.

MARTÍNEZ-ESPINOSA, M.; LOUZADA-NETO, F.; CALIL JÚNIOR, C. Estatística geral com aplicações à engenharia. São Paulo: Atlas, 2002.

ORMROD, J. E. Predictors of faculty dissastisfaction with na annual performance evaluation. CUPA. Journal, 37, 13-17. Tradução de Paulo Henrique Britto. 1986.

POLIT, D. F.; BECK, C. T.; HUNGLER, B. P. Fundamentos de pesquisa em enfermagem: métodos, avaliação e utilização. Trad. de Ana Thorell. 5. ed. Porto Alegre:Artmed, 2004.

REIFSCHNEIDER, M. B. Considerações sobre avaliação de desempenho. Ensaio: avaliação políticas públicas Educação. Rio de Janeiro, v. 16, n. 58, p. 47-58, jan./mar. 2008. Disponível em: http://www.scielo.br/pdf/ensaio/v16n58/a04v1658.pdf Acesso em: 06.05.2014.

RHODES, F.H.T. The new American university.David Dodds Henry Series, University of Illinois. 1990.

UNIVERSIDADE DO VALE DO ITAJAÍ. Perfil dos Estudantes de Graduação da Universidade do Vale do Itajaí. Itajaí: UNIVALI, 2005.

. A avaliação institucional na Univali 1993-2009. Caderno de ensino. Documentos Institucionais; Ano 9, n. 121. Itajaí, 2010. 